\title{
Influence of Vibrotactile Random Noise on the Smoothness of the Grasp Movement in Patients in Chemotherapy-Induced Peripheral Neuropathy
}

Michihiro Osumi ( $\sim$ m.ohsumi@kio.ac.jp )

Kio University

Masahiko Sumitani

The University of Tokyo Hospital

Yuko Otake

Tohto University

Yuki Nishi

Kio University

Satoshi Nobusako

Kio University

Shu Morioka

Kio University

\section{Research Article}

Keywords: Random noise, Stochastic resonance, Chemotherapy-induced peripheral neuropathy, Grasp movement

Posted Date: December 20th, 2021

DOI: https://doi.org/10.21203/rs.3.rs-1160733/v1

License: (9) This work is licensed under a Creative Commons Attribution 4.0 International License. Read Full License 


\section{Abstract}

Background Patients with chemotherapy-induced peripheral neuropathy (CIPN) often suffer from sensorimotor dysfunction of the distal portion of the extremities (e.g., loss of somatosensory sensation, numbness/tingling, difficulty typing on a keyboard, or difficulty undoing or doing up a button). The present study aimed to reveal the effects of subthreshold vibrotactile random noise stimulation on sensorimotor dysfunction in CIPN patients without exacerbating symptoms.

Methods Twenty-five patients with CIPN and 28 age-matched healthy adults participated in this study. To reveal the effects of subthreshold vibrotactile random noise stimulation on sensorimotor function, participants were asked to perform grasp movement tasks during random noise stimulation delivered to the volar and dorsal wrist. We set three intensity conditions of the vibrotactile random noise: $0 \%, 60 \%$, and $120 \%$ of the sensory threshold (Noise $0 \%$, Noise $60 \%$, and Noise $120 \%$ conditions). In the grasp movement task, the distance between the thumb and index finger was recorded while the participant attempted to grasp a target object, and the smoothness of the grasp movement was quantified by calculating normalized jerk in each experimental condition. The experimental data were compared using two-way repeated-measures analyses of variance with two binary factors: experimental condition (Noise $0 \%, 60 \%, 120 \%) \times$ group (Healthy controls, CIPN patients) .

Results The smoothness of the grasp movement was only improved in the Noise $60 \%$ condition without exacerbating numbness/tingling in CIPN patients and healthy controls.

Conclusions The current study suggested that the development of treatment devices using stochastic resonance can improve sensorimotor function for CIPN patients.

Trial registration UMIN Clinical Trials Registry UUMIN-CTR囚No. UMIN000024776

https://rctportal.niph.go.jp/s/detail/um?trial_id=UMIN000024776\#

\section{Background}

Cancer is a major contributor to the global disease burden. The number of long-term cancer survivors is increasing because of improvements in early diagnosis and treatment [1]. Mitigating disability associated with the long-term effects of cancer treatment is important for improving quality of life (QOL) in cancer survivors. Chemotherapy-induced peripheral neuropathy (CIPN) is one of the most common complications of cancer treatments, with an incidence of $30 \%-70 \%$ in cancer patients receiving chemotherapy [2]. CIPN typically persists beyond several years after initiating chemotherapy. [3]. Most patients with CIPN suffer from somatosensory symptoms (e.g., loss of somatosensory, numbness/tingling, and neuropathic pain) in the distal portion of the extremities, which can spread to the stocking-glove pattern. These somatosensory symptoms can directly affect precise finger movements in daily living (e.g., typing on a keyboard, undoing and doing up buttons) [4]. In a previous study, we kinematically quantified precise finger movements using a three-dimensional measurement system and 
revealed impaired smoothness of thumb-index grasp movements in CIPN patients [5]. Several studies reported that exercise programs including resistance training, balance training, and cardiovascular exercise provided benefits for patients suffering from CIPN [6, 7]. Although simple exercise programs have been found to improve disability and QOL, they have demonstrated limited efficacy on somatosensory dysfunction derived from CIPN [8]. In addition, there is little evidence regarding the efficacy of physical and occupational therapies for precise finger movements, although these are recommended for improving disability and QOL [9]. Therefore, novel treatment strategies may be useful for improving somatosensory dysfunction and subsequent precise finger movements in patients with CIPN.

Somatosensory inputs from the periphery are thought to play an important role in recovering precise movements after improving somatosensory function [10]. For example, suprathreshold electrical stimulation in the affected upper extremity has been reported to improve paralysis [11]. Considering such suprathreshold somatosensory stimulation causes discomfort and a tingling sensation [12], suprathreshold somatosensory stimulation might exacerbate numbness and allodynia in CIPN patients. To compensate for such adverse effects, we alternatively focused on subthreshold somatosensory stimulation to improve somatosensory function, which is known as the stochastic resonance phenomenon. In the stochastic resonance phenomenon, subthreshold vibrotactile random noise stimulation applied to the wrist and ankle has been reported to improve both the tactile detection thresholds of other somatosensory stimuli and feedback motor control in the hand and foot, respectively [13]. Clinical application of subthreshold random noise stimulation was reported to improve the somatosensory sensitivity and motor function of the affected limbs in older adults and patients with diabetic peripheral neuropathy or stroke [14-18]. The present study aimed to investigate the clinical usefulness of subthreshold random noise stimulation for treating impaired somatosensory function and precise movements in CIPN patients.

\section{Methods}

\section{Participants}

Twenty-five patients with CIPN (age, $64.8 \pm 9.4$, mean \pm standard deviation; 15 females) and 28 agematched adults with no hand-related problems (age, $65.8 \pm 9.5$; 16 females) participated in this study. Most CIPN patients reported numbness, neuropathic pain, and difficulties with precise hand movements after receiving chemotherapy. Clinical assessments of CIPN were conducted using part of the FACT/GOG NTX Ver.4.0 (https://www.facit.org/measures/FACT-GOG-NTX), the Patient Neurotoxicity Questionnaire (PNQ), the Japanese version of the Neuropathic Pain Symptom Inventory (NPSI) [19], and an 11-point numerical rating scale. The FACT/GOG-NTX Ver.4.0 measured symptoms of peripheral neuropathy, including sensory and motor problems, with a 5-point Likert scale $(0=$ Not at all, $1=$ A little bit, $2=$ Somewhat, 3 = Quite a bit, 4 = Very much). Among items of the FACT/GOG-NTX Ver.4.0, we used the NTX subscale comprising 11 items concerning neurotoxicity (NTX1, NTX2, NTX3, NTX4, NTX5, NTX 6, NTX 7, NTX 8, NTX 9, HI12, An6) [20,21]. The PNQ includes two questionnaire items: one asking about sensory 
neurotoxicity and the other asking about motor neurotoxicity. Answers were coded 0 to 4 , with a higher score indicating more severe CIPN. The NPSI is a self-administered questionnaire specifically designed to evaluate the different positive and negative symptoms of neuropathic pain. The NPSI comprises 12 items to identify the presence of respective neuropathic pain sensations on an 11-point (0-10) numerical scale, in which the total score represents the scores from 10 items. In addition, pain intensity was assessed using a numerical rating scale in which the patient was asked to grade the actual pain level experienced on a scale from $0-10(0=$ no pain, $10=$ worst pain imaginable $)$. The ethical review board of our institution approved this study (Approval Number: H28-42). We explained the protocol of this study to all participants, and obtained written informed consent.

\section{Vibrotactile Random Noise Stimulation}

Vibrotactile random noise stimulation was applied using two small actuators (length: $10 \mathrm{~mm}$; width: 18 mm; height: 2 mm; Vibration Actuator Sprinter a; Nidec Seimitsu, Nagano, Japan) attached to the volar and dorsal wrist using adhesive tape. White noise signals that were low-pass filtered at $500 \mathrm{~Hz}$ drove the actuators, as described in a previous study [22]. The intensity levels of the vibrotactile random stimulation were set to $60 \%$ (Noise $60 \%$ condition) or $120 \%$ (Noise $120 \%$ condition) of the somatosensory detection threshold, which was defined at the beginning of this study. In the baseline condition, no tactile random noise stimulation was presented (Noise $0 \%$ condition). All participants performed a tactile detection task and a grasp movement task while receiving each level of vibrotactile stimulation intensity (Noise $0 \%, 60 \%$, and $120 \%$ conditions), as described below.

\section{Tactile detection task}

Participants performed the tactile detection task using a Semmes-Weinstein monofilament (log of force: 1.65-6.65) based on standard testing measures [23]. We applied each filament until it bent onto the volar surface of the index-fingertip at least five times, in an ascending fashion to minimize participants' fatigue. Starting with a 2.36 filament as the baseline, the evaluation was continued using filaments of increasing weight until participants reported perceiving a touch, or until 6.65 was reached. We interpreted the tactile detection threshold as the force value of the filament weight that participants could first perceive in each experimental condition.

\section{Grasp movement task}

The grasp movement task assessed the precise movements of the hand. Participants were asked to grasp a target object ( $5 \mathrm{~mm}$ diameter, $35 \mathrm{~mm}$ height) located $30 \mathrm{~cm}$ away from the starting position (Fig. 1). Participants conducted this task at an arbitrary speed and repeated 10 trials with each vibrotactile random noise stimulation. To record the grasp movements, magnetic sensors were attached to the tips of the thumb and the index finger, and the distance (i.e., aperture length) between the two sensors was extracted using a sampling rate of $100 \mathrm{~Hz}$ (UB-2, Maxell, Ltd. Tokyo) [24]. Using the data for the aperture length, the smoothness of the grasping movements was quantified using normalized jerk (NJ) measurement. In NJ measurement, jerk is mathematically defined as the third time derivative of the 
aperture length variable, and low values indicate smooth movement [25]. The jerk value depends on the movement length $(L)$ squared divided by the fifth power of movement duration $(M D), L^{2} / M D^{5}$, even when the shape of the grasp aperture curve is invariant $[25,26]$. Therefore, grasp jerk needs to be normalized. $\mathrm{NJ}_{\text {grasp }}$ was calculated using the following equations, which normalized the dependence on the movement duration and length. In these equations, MD represents the time between the start and end of the movement. In this study, $\mathrm{L}_{\text {grasp }}$ represented the difference in grasp aperture between the start and end of the movement. The $\mathrm{NJ}_{\text {grasp }}$ values were log-transformed to meet the assumptions of normality [26].

$$
N J_{\text {grasp }}=\sqrt{\frac{1}{2} \int_{t(\text { start })}^{t(e n d)} j e r k_{\text {grasp }}^{2}(\mathrm{t}) \mathrm{dt} * M D^{5} / L_{\text {grasp }}^{2}}
$$

\section{Statistical analysis}

First, to verify sensorimotor dysfunction in CIPN, patients' tactile detection threshold and log of NJ in the Noise $0 \%$ condition as the baseline were compared with those of age-matched controls using the MannWhitney $U$ test. Second, to identify the effect of the vibrotactile random noise stimulation, the experimental data were compared using a two-way repeated-measures analysis of variance (ANOVA) for two binary factors: condition (Noise $0,60,120 \%) \times$ group (health controls, CIPN patients). The Bonferroni method was used for post hoc testing. A difference was considered significant at $p<0.05$.

\section{Results}

\section{Tactile detection threshold}

The baseline tactile detection threshold in CIPN patients was higher than that in controls $(U=203.0, p=$ 0.009). No patients complained of worsening pain or numbness while undergoing each experimental condition. Two-way repeated-measures ANOVA revealed significant main effects of group $(U=239.0, p=$ 0.03 ) and condition $(F=24.05, p<0.001)$. There was no significant interaction effect between group and intervention session $(F=0.148, p=0.812)$. Post hoc tests revealed that the tactile detection threshold in controls in the Noise $60 \%$ condition was significantly lower than that in the Noise $0 \%$ condition $(t=3.69, p$ $=0.001)$ and the Noise $120 \%$ condition $(t=-3.76, p=0.01)$. The tactile detection threshold in CIPN patients in the Noise $60 \%$ condition was also significantly lower than that in the Noise $0 \%$ condition $(t=$ $3.91, p=0.001)$ and Noise $120 \%$ condition $(t=-3.88, p=0.001)$.

\section{$\mathrm{NJ}$ of grasp movement}

The log of the NJ in CIPN patients was significantly higher than that in controls $(t=-2.99, p=0.004)$. Two-way repeated-measures ANOVA revealed significant main effects of group $(F=14.02, p<0.001)$ and condition $(F=16.61, p<0.001)$. There was no significant interaction effect between group and intervention session $(F=0.001, p=0.97)$. Post hoc tests revealed that the log of $N J$ in controls in the 
Noise $60 \%$ condition was lower than that in the Noise $0 \%$ condition $(t=3.15, p=0.01)$ and Noise $120 \%$ condition $(t=-2.99, p=0.01)$. The log of the $\mathrm{NJ}$ in CIPN patients in the Noise $60 \%$ condition was also significantly lower than that in the Noise $0 \%$ condition $(t=4.65, p<0.001)$ and Noise $120 \%$ condition $(t=$ $-3.64, p<0.001)$.

\section{Discussion}

The present study demonstrated that the tactile detection threshold and NJ values of grasp movements in CIPN patients were higher than those in age-matched controls. The findings quantitatively indicated that somatosensory function and precise finger movements of CIPN patients were profoundly impaired.

The impaired tactile detection threshold and smoothness of grasping movements in CIPN significantly improved when vibrotactile random noise stimulation was applied with $60 \%$ intensity of the tactile detection threshold, but not when stimulation was applied at $120 \%$ or $0 \%$ of the threshold. Thus, the results indicated that the stochastic resonance phenomenon (i.e., subthreshold stimulation) was effective for improving somatosensory dysfunction and dysfunction of precise movements in patients with CIPN. In addition, the present findings are in agreement with a previous report that subthreshold random noise stimulation improved the sensory-motor system in patients with stroke [27]. As previously reported in patients with somatosensory disorder (i.e., post-stroke sensory ataxia) who exhibited impaired jerk during grasp movements, precise grasp movements require sensorimotor coordination using visual and somatosensory information [28]. Thus, the improvement of grasping movements in the stochastic resonance condition in the current study suggests that subthreshold random noise stimulation may have congruently adjusted the sensory-motor system. A previous study using electroencephalography provides anecdotal support for the suggestion that the subthreshold random noise stimulation enhances sensorimotor cortical activity [18].

As an alternative to the present subthreshold somatosensory stimulation, several previous studies reported improved sensory and motor symptoms of CIPN patients using suprathreshold sensory stimulation (e.g., cold stimulation, transcutaneous electrical nerve stimulation, vibratory stimulation) [29, $30,31]$. In one study, an extensive approach combining suprathreshold whole-body vibration stimulation with standard physical therapy was applied for symptoms of CIPN [30]. However, a clinical study reported that applying suprathreshold somatosensory stimulation to the affected hands and feet of CIPN patients can exacerbate sensations of numbness and discomfort [32]. In contrast, subthreshold vibrotactile random noise stimulation is not perceived when applied, potentially minimizing the risk of exacerbating numbness and allodynia, both of which are characteristic symptoms of CIPN. Thus, even if subthreshold random noise stimulation was applied over a long period, CIPN patients would not be expected to complain of sensations of discomfort and annoyance.

\section{Conclusion}


The "silent" nature of subthreshold random noise stimulation may enable patients to keep applying the stimulation in their daily lives and consequently improve the smoothness of specific movements, such as typing on a keyboard and undoing and doing up buttons, ultimately improving their QOL. Moreover, because subthreshold random noise can enhance motor skill learning [33], application of subthreshold noise stimulation might improve the effects of physical and occupational therapy.

\section{Abbreviations}

CIPN: Chemotherapy-induced peripheral neuropathy

QOL: Quality of life

PNQ: Patient Neurotoxicity Questionnaire

NPSI: Neuropathic Pain Symptom Inventory

\section{Declarations}

Ethics approval and consent to participate: The ethical review board of our institution approved this study (Approval Number: H28-42). We explained the protocol of this study to all participants, and obtained written informed consent.

Consent for publication: Not applicable.

Availability of data and materials: The datasets used and/or analyzed during the current study are available from the corresponding author on reasonable request.

Competing interests: The authors declare that they have no competing interests.

Funding: This study was supported by a grant from JSPS KAKENHI Grant Numbers 17K13080.

Authors' contributions: $\mathrm{MO}$ and $\mathrm{YO}$ and $\mathrm{YN}$ recorded and analyzed the patient's data. MO and MS were major contributor in writing the manuscript. SN supported statistical processing. SM supervised the study. All authors read and approved the final manuscript.

Acknowledgements: We thank Benjamin Knight, MSc., from Edanz (https://jp.edanz.com/ac) for editing a draft of this manuscript, and Dr Atsushi Matsuo for providing many advises about neurorehabilitation.

\section{Authors' information (optional):}

Japanese Physical Therapy Association

Michihiro Osumi, Yuko Otake, Yuki Nishi, Satoshi Nobusako, Shu Morioka

Footnotes: Not applicable. 


\section{References}

1. Siegel RL, Miller KD, Jemal A. Cancer statistics, 2019. CA Cancer J Clin. 2019;69(1):7-34.

2. Cavaletti G, Marmiroli P. Chemotherapy-induced peripheral neurotoxicity. Nat Rev Neurol. 2010;6:657e666.

3. Tanabe Y, Hashimoto K, Shimizu C, Hirakawa A, Harano K, Yunokawa M, et al. Paclitaxel-induced peripheral neuropathy in patients receiving adjuvant chemotherapy for breast cancer. Int $\mathrm{J}$ Clin Oncol. 2013;18(1):132-138.

4. Gentilucci M, Toni I, Daprati E, Gangitano M. Tactile input of the hand and the control of reaching to grasp movements. Exp Brain Res. 1997;114(1):130-137.

5. Osumi M, Sumitani M, Abe H, Otake Y, Kumagaya SI, Morioka S. Kinematic evaluation for impairment of skilled hand function in chemotherapy-induced peripheral neuropathy. J Hand Ther. 2019;32(1):41-47.

6. McCrary JM, Goldstein D, Sandler CX, Barry BK, Marthick M, Timmins HC, et al. Exercise-based rehabilitation for cancer survivors with chemotherapy-induced peripheral neuropathy. Support Care Cancer. 2019;27(10):3849-3857.

7. Andersen Hammond E, Pitz M, Steinfeld K, Lambert P, Shay B. An Exploratory Randomized Trial of Physical Therapy for the Treatment of Chemotherapy-Induced Peripheral Neuropathy. Neurorehabil Neural Repair. 2020;34(3):235-246.

8. Bland KA, Kirkham AA, Bovard J, Shenkier T, Zucker D, McKenzie DC, et al. Effect of Exercise on Taxane Chemotherapy-Induced Peripheral Neuropathy in Women With Breast Cancer: A Randomized Controlled Trial. Clin Breast Cancer. 2019;19(6):411-422.

9. Kim JH, Dougherty PM, Abdi S. Basic science and clinical management of painful and non-painful chemotherapy-related neuropathy. Gynecol Oncol. 2015;136(3):453-459.

10. Bolognini N, Russo C, Edwards DJ. The sensory side of post-stroke motor rehabilitation. Restor Neurol Neurosci. 2016;34(4):571-586.

11. Conforto AB, Dos Anjos SM, Bernardo WM, Silva AAD, Conti J, Machado AG, et al. Repetitive Peripheral Sensory Stimulation and Upper Limb Performance in Stroke: A Systematic Review and Meta-analysis. Neurorehabil Neural Repair. 2018;32(10):863-871.

12. Golaszewski SM, Siedentopf CM, Koppelstaetter F, Rhomberg P, Guendisch GM, Schlager A, et al. Modulatory effects on human sensorimotor cortex by whole-hand afferent electrical stimulation. Neurology. 2004;62(12):2262-2269.

13. Collins JJ, Imhoff TT, Grigg P. Noise-enhanced tactile sensation. Nature. 1996;383(6603):770.

14. Collins JJ. Noise-enhanced vibrotactile sensitivity in older adults, patients with stroke, and patients with diabetic neuropathy. Arch Phys Med Rehabil. 2002;83(2):171-176.

15. Wells C, Ward LM, Chua R, Timothy Inglis J. Touch noise increases vibrotactile sensitivity in old and young. Psychol Sci. 2005;16(4):313-320. 
16. Enders LR, Hur P, Johnson MJ, Seo NJ. Remote vibrotactile noise improves light touch sensation in stroke survivors' fingertips via stochastic resonance. J Neuroeng Rehabil. 2013;10:105.

17. Lakshminarayanan K, Lauer AW, Ramakrishnan V, Webster JG, Seo NJ. Application of vibration to wrist and hand skin affects fingertip tactile sensation. Physiol Rep. 2015;3(7):e12465.

18. Seo NJ, Lakshminarayanan K, Lauer AW, Ramakrishnan V, Schmit BD, Hanlon CA, et al. Use of imperceptible wrist vibration to modulate sensorimotor cortical activity. Exp Brain Res. 2019;237(3):805-816.

19. Matsubayashi Y, Takeshita K, Sumitani M, Oshima Y, Tonosu J, Kato S, et al. Psychometric Validation of the Japanese Version of the Neuropathic Pain Symptom Inventory. PLoS One. 2015;10(11):e0143350.

20. Calhoun EA, Welshman EE, Chang CH, Lurain JR, Fishman DA, Hunt TL, et al. Psychometric evaluation of the Functional Assessment of Cancer Therapy/Gynecologic Oncology GroupNeurotoxicity (Fact/GOG-Ntx) questionnaire for patients receiving systemic chemotherapy. Int J Gynecol Cancer. 2003;13(6):741-748.

21. Huang HQ, Brady MF, Cella D, Fleming G. Validation and reduction of FACT/GOG-Ntx subscale for platinum/paclitaxel-induced neurologic symptoms: a gynecologic oncology group study. Int J Gynecol Cancer. 2007;17(2):387-393.

22. Enders LR, Hur P, Johnson MJ, Seo NJ. Remote vibrotactile noise improves light touch sensation in stroke survivors' fingertips via stochastic resonance. J Neuroeng Rehabil. 2013;10:105.

23. Bell-Krotoski J, Weinstein S, Weinstein C. Testing sensibility, including touch-pressure, two-point discrimination, point localization, and vibration. J Hand Ther. 1993;6(2):114-123.

24. Sano Y, Kandori A, Shima K, Yamaguchi Y, Tsuji T, Noda M, et al. Quantifying Parkinson's disease finger-tapping severity by extracting and synthesizing finger motion properties. Med Biol Eng Comput. 2016;54(6):953-965.

25. Hogan N, Sternad D. Sensitivity of smoothness measures to movement duration, amplitude, and arrests. J Mot Behav. 2009;41(6):529-534.

26. van Kordelaar J, van Wegen E, Kwakkel G. Impact of time on quality of motor control of the paretic upper limb after stroke. Arch Phys Med Rehabil. 2014;95(2):338-344.

27. Seo NJ, Kosmopoulos ML, Enders LR, Hur P. Effect of remote sensory noise on hand function post stroke. Front Hum Neurosci. 2014;8:934.

28. Gentilucci M, Daprati E, Gangitano M. Haptic information differentially interferes with visual analysis in reaching-grasping control and in perceptual processes. Neuroreport. 1998;9(5):887-891.

29. Bailey AG, Brown JN, Hammond JM. Cryotherapy for the prevention of chemotherapy-induced peripheral neuropathy: A systematic review. J Oncol Pharm Pract. 2021;27(1):156-164.

30. Schönsteiner SS, Bauder Mißbach H, Benner A, Mack S, Hamel T, et al. A randomized exploratory phase 2 study in patients with chemotherapy-related peripheral neuropathy evaluating whole-body vibration training as adjunct to an integrated program including massage, passive mobilization and physical exercises. Exp Hematol Oncol. 2017;6:5. 
31. Childs DS, Le-Rademacher JG, McMurray R, Bendel M, O'Neill C, Smith TJ, et al. Randomized Trial of Scrambler Therapy for Chemotherapy-Induced Peripheral Neuropathy: Crossover Analysis. J Pain Symptom Manage. 2021;61(6):1247-1253.

32. Şimşek NY, Demir A. Cold Application and Exercise on Development of Peripheral Neuropathy during Taxane Chemotherapy in Breast Cancer Patients: A Randomized Controlled Trial. Asia Pac J Oncol Nurs. 2021;8(3):255-266.

33. Prichard G, Weiller C, Fritsch B, Reis J. Effects of different electrical brain stimulation protocols on subcomponents of motor skill learning. Brain Stimul. 2014;7(4):532-540.

\section{Figures}

\section{Healthy controls}

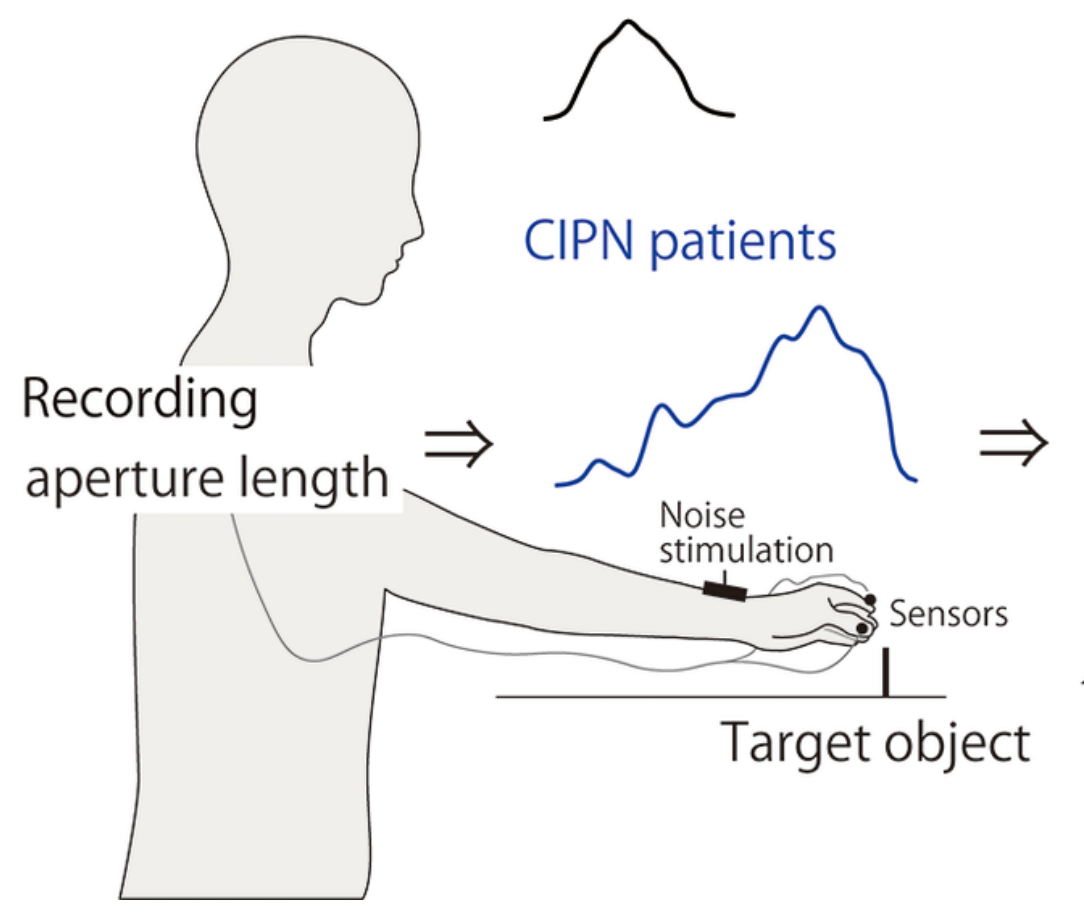

\section{Figure 1}

Experimental setup of the kinematic recording and evaluating normalized jerk of grasp movements. Example of time series of aperture length in age-matched controls (black) and CIPN patients (blue). 

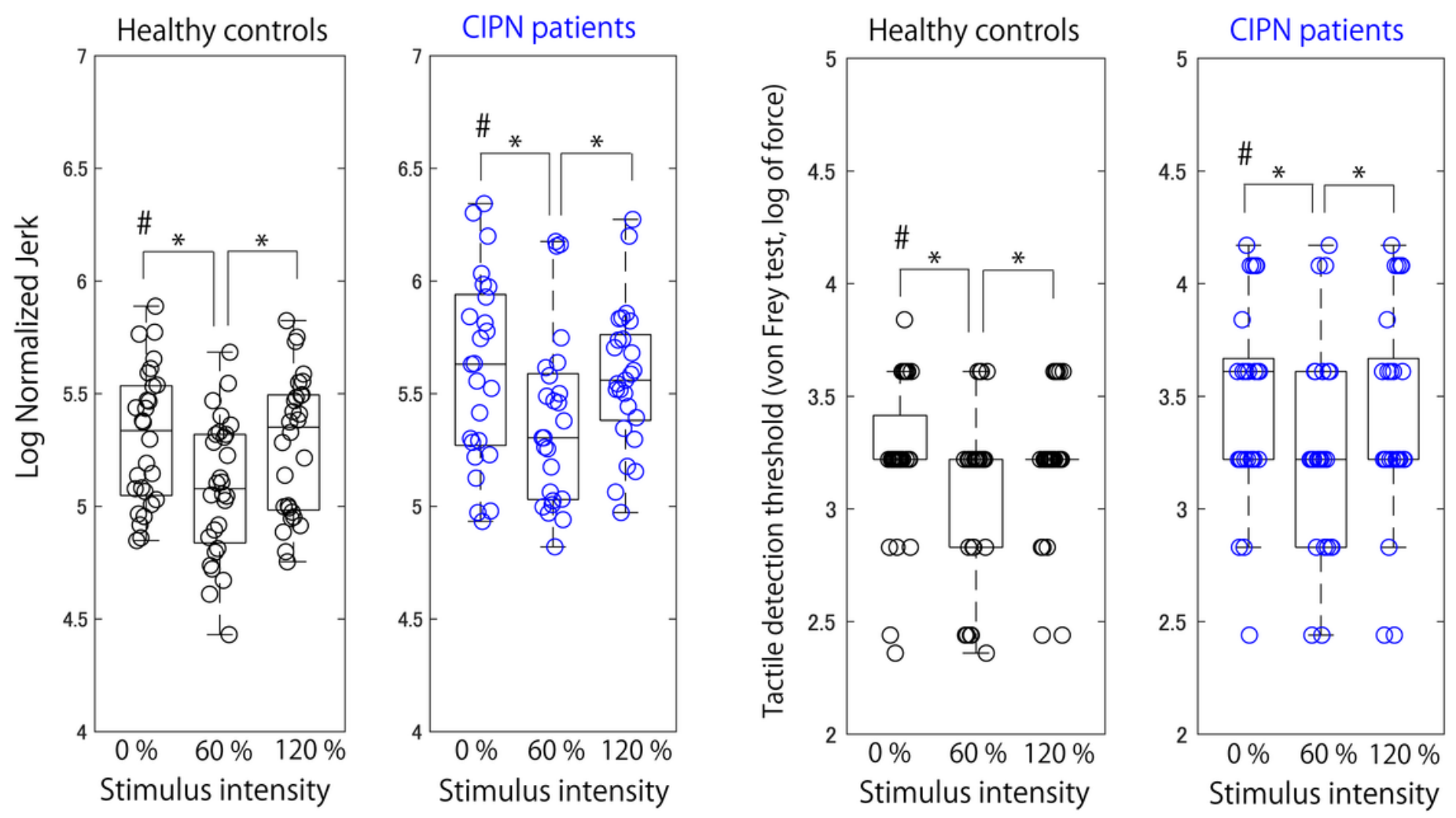

Figure 2

Comparisons of the log normalized jerk of grasp movement and tactile detection threshold between three stimulus conditions (Noise $0 \%, 60 \%$, and $120 \%$ condition). *Significant difference between stimulus conditions.

\#Significant difference between healthy controls and CIPN patients in the Noise $0 \%$ condition. 